\title{
Contact Relation Algebras
}

\author{
Ivo Düntsch \\ School of Information and Software Engineering \\ University of Ulster at Jordanstown \\ Newtownabbey, BT 37 0QB, N.Ireland \\ I.Duentsch@ulst.ac.uk
}

\begin{abstract}
Contact relation algebras (CRAs), introduced in Düntsch et al. (1999), arise from the study of "part-of" and "contact" relations rooted in mereology and have applications, for example, in qualitative spatial reasoning. We give an overview of the origins of CRAs and numerous examples.
\end{abstract}

\section{Introduction}

Contemporary qualitative spatial reasoning (QSR) is based largely on the relational and topological properties of regions. A basic role is played by the binary "part - of" and "contact" relations, from which many others can be defined. The formal study of "part - of" relations goes back to S. Leśniewski (1927 - 1931), a Polish mathematician, who, together with Twardowsky, Łukasiewicz, and his sole doctoral student Tarski, formed the core of the Lwów - Warsaw school of Logic and Philosophy, which

"... in the 20s -30 s of this century made the University of Warsaw perhaps the most important research centre in the world for formal logic" (Betti, 1997).

Mereology ${ }^{1}$, the "Science of parts", is a part of S. Leśniewski's work on the foundations of Mathematics, developed from about 1915 onwards (Leśniewski, 1927 - 1931). It is not the purpose of this article to go into the details and ramifications of this system, but we refer the reader instead to Leśniewski (1983), Luschei (1962), Surma et al. (1992) or Link (1998), Simons (1987). Mereology was later taken up by Leonard \& Goodman (1940), though for a different reason; formally, their calculus and Leśniewski's system are the same. Clarke (1981) generalised mereology and based the relational part of his "Calculus of Individuals" on a "connection" or "contact" relation which first appeared in the works of de Laguna (1922) and Nicod (1924). In spatial reasoning, "mereology" is today frequently

\footnotetext{
${ }^{1}$ Tò $\mu \varepsilon ́ p \varepsilon o \varsigma=$ The part
} 
used synonymously with the study of connection, and Leśniewski's original system is referred to as “classical mereology" (CM) (see Eschenbach \& Heydrich, 1995).

A "connection" or "contact" relation is a reflexive and symmetric binary relation with the additional property that each element of the domain is determined by those elements with which it is in contact. From a contact relation between circles in the Euclidean plane the relations pictured in Figure 2 on page 6 can be defined citeplag22,cla81. These relations also form the basis of the investigations of Egenhofer \& Franzosa (1991) and Randell et al. (1992).

Recently, composition based reasoning with binary relations has been of interest to the QSR community (Bennett, 1997, 1998, Bennett et al., 1997, Randell et al., 1992), and the expressive power, consistency and complexity of relational reasoning have become an object of study.

It has been known for some time, that the expressiveness of reasoning with basic operations on binary relations is equal to the expressive power of the three variable fragment of first order logic (see Tarski \& Givant, 1987, and the references therein). Thus, it seems worthwhile to use methods of relation algebras, initiated by Tarski (1941), to study contact relations in their own right, and then explore their expressive power with respect to various topological domains.

In this paper, we give an overview of the basic properties of contact relations and their algebras, and explore their expressive power on simple domains; most of the material is drawn from Düntsch et al. $(1999,2001 b)$.

\section{Binary relations and their algebras}

A binary relation on a set $U$ is a subset of $U \times U$. If $R, S \subseteq U \times U$, and $x, y, z \in U$, we will usually write $x R y$ for $\langle x, y\rangle \in R$, and $x R y S z$ for $x R y$ and $y R z$. The range of $x$ in $R$ is the set

$$
R x \stackrel{\text { def }}{=}\{y \in U: x R y\} .
$$

We denote the set of all binary relations on $U$ by $\operatorname{Rel}(U)$; clearly, $\operatorname{Rel}(U)$ is a Boolean algebra under the usual set operations $\cap, \cup,-$ with smallest element $\emptyset$ and largest element $V \stackrel{\text { def }}{=} U \times U$. We also consider the following operations on $\operatorname{Rel}(U)$ :

$$
\begin{array}{rlrl}
R \circ S & \stackrel{\text { def }}{=}\{\langle x, y\rangle:(\exists z \in U)[x R z S y]\}, & & \text { Composition } \\
R \stackrel{\text { def }}{=}\{\langle x, y\rangle: y R x\} . & & \text { Converse }
\end{array}
$$

An additional distinguished constant is the identity relation $1^{\prime}$. The structure

$$
\left\langle\operatorname{Rel}(U), \cap, \cup,-, \emptyset, V, \circ, \sim, 1^{\prime}\right\rangle
$$

is called the full algebra of binary relations on $U$. Any subalgebra of $\operatorname{Rel}(U)$ is called an algebra of binary relations (BRA). We usually identify algebras with their base set and write $A \leq \operatorname{Rel}(U)$ if $A$ 
is a subalgebra of $\operatorname{Rel}(U) . A \leq \operatorname{Rel}(U)$ is called integral, if $1^{\prime}$ is an atom of $A$. If $\left\{R_{i}: i \in I\right\} \subseteq$ $\operatorname{Rel}(U)$, then $\left\langle R_{i}\right\rangle_{i \in I}$ is the subalgebra of $\operatorname{Rel}(U)$ generated by $\left\{R_{i}: i \in I\right\}$.

If $R \in \operatorname{Rel}(U)$, then its residual $R \backslash R$ is the largest $S \in \operatorname{Rel}(U)$ for which $R \circ S \subseteq R$. The residual is equationally definable as

$$
R \backslash R=-\left(R^{\triangleleft} \circ-R\right)
$$

and one can also show that

$$
x(R \backslash R) y \Longleftrightarrow R x \subseteq R y .
$$

Furthermore,

Lemma 2.1. (Pratt, 1990) $R \backslash R$ is reflexive and transitive.

The expressiveness of BRAs corresponds to a fragment of first order logic, and the following fundamental result is due to A. Tarski (see Tarski \& Givant, 1987):

Theorem 2.2. If $R_{0}, \ldots, R_{k} \in \operatorname{Rel}(U)$, then $\left\langle R_{0}, \ldots, R_{k}\right\rangle$ is the set of all binary relations on $U$ which are definable in the (language of the) relational structure $\left\langle U, R_{0}, \ldots, R_{k}\right\rangle$ by first order formulas using at most three variables, two of which are free.

In certain domains, BRA expressiveness is even stronger: We call a BRA $A \leq \operatorname{Rel}(U)$ first order closed, if every relation definable in the first order structure $\langle U, A\rangle$ is an element of $A$.

If a BRA $A \leq \operatorname{Rel}(U)$ is complete and atomic - in particular, if $A$ is finite -, then each nonzero element is a sum of atoms, and relational composition can be be described by a matrix such as Table 1 , whose rows and columns are labelled by the atoms and an entry $\langle P, Q\rangle$ is the set of atoms contained in $P \circ Q$. If $A$ is integral, we omit column and row $1^{\prime}$.

For example, the algebra determined by Table 1 has the atoms $P P, P P^{\sim}, P O, D C, 1^{\prime}$; we see that $P O$ and $D C$ are symmetric relations, and that $1^{\prime}$ is an atom. The entry, for example, at position $\left\langle P P^{\sim}, P P\right\rangle$ means that

$$
P P^{\cup} \circ P P=-D C=P P \cup P P^{\cup} \cup P O \cup 1^{\prime} .
$$

If $\mathcal{R}=\left\{R_{i}: i \in I\right\}$ is a partition of $\operatorname{Rel}(U)$ such that $\mathcal{R}$ is closed under converse, and $R_{i} \subseteq 1^{\prime}$ or $R_{i} \cap 1^{\prime}=\emptyset$ for all $i \in I$, we define the weak composition of $\mathcal{R}$ as the mapping $\circ_{w}: \mathcal{R} \times \mathcal{R} \rightarrow 2^{\mathcal{R}}$ such that for all $i, j \in I$

$$
S \in R_{i} \circ R_{j} \stackrel{\text { def }}{\Longleftrightarrow} S \cap\left(R_{i} \circ R_{j}\right) \neq \emptyset .
$$

Just as in the case of o, we can determine composition tables for $\circ_{w}$. Note that $R_{i} \circ R_{j} \subseteq R_{i} \circ_{w} R_{j}$; if equality holds everywhere, i.e. when $\circ=o_{w}$, we call the weak composition table extensional. 
Table 1: Open circle algebra $\mathcal{C}_{o}$

\begin{tabular}{|c||c|c|c|c|}
\hline$\circ$ & $P P$ & $P P^{\backsim}$ & $P O$ & $D C$ \\
\hline \hline$P P$ & $P P$ & $V$ & $-P^{\sim}$ & $D C$ \\
\hline$P P^{\sim}$ & $-D C$ & $P P^{\triangleleft}$ & $P P^{\sim}, P O$ & $-P$ \\
\hline$P O$ & $P P, P O$ & $-P$ & $V$ & $-P$ \\
\hline$D C$ & $-P^{\sim}$ & $D C$ & $-P^{\backsim}$ & $V$ \\
\hline
\end{tabular}

An abstract relation algebra (RA) is a structure

$$
\left\langle A,+, \cdot,-, 0,1, \circ,^{\lrcorner}, 1^{\prime}\right\rangle
$$

of type $\langle 2,2,1,0,0,2,1,0\rangle$ which satisfies for all $a, b, c \in A$,

1. $\langle A,+, \cdot,-, 0,1\rangle$ is a Boolean algebra (BA).

2. $\left\langle A, \circ,{ }^{\prime}, 1^{\prime}\right\rangle$ is an involuted monoid, i.e.

(a) $\left\langle A, \circ, 1^{\prime}\right\rangle$ is a semigroup with identity $1^{\prime}$,

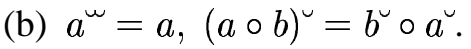

3. The following conditions are equivalent:

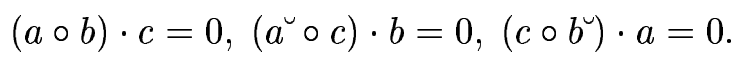

Each BRA is an RA with the obvious operations, but not vice versa (Lyndon, 1950). If $A$ is an abstract $\mathrm{RA}$, a representation of $A$ is a BRA isomorphic to $A .^{2}$

For the arithmetic and other properties of BRAs we invite the reader to consult Chin \& Tarski (1951) or Jónsson $(1982,1991)$.

\section{Relations of time and space}

Allen (1983) has presented a set of 13 relations which characterise the possible relations between convex intervals of time. ${ }^{3}$ These are the six relations of Table 2 , their converses, and the identity. They are the atoms of an integral BRA on the set of all closed intervals on the real line; its composition table can be found in e.g. Ladkin \& Maddux (1994). We observe that, in this model, the basic object in the ontology of time is the interval, as opposed to a point, and we invite the reader to consult Allen (1984) for a discussion of this issue.

\footnotetext{
${ }^{2}$ This is more special than the usual definition, but it will suffice for our purposes.

${ }^{3} \mathrm{~A}$ logic of time was independently given by van Benthem (1983), see also Nicod (1924).
} 
Table 2: Interval relations

\begin{tabular}{|r|}
\hline before: $\left\{\left\langle[q, r],\left[q^{\prime}, r^{\prime}\right]\right\rangle: q<r<q^{\prime}<r^{\prime}, q, r, q^{\prime}, r^{\prime} \in \mathbb{R}\right\}$ \\
\hline meets: $\left\{\left\langle[q, r],\left[q^{\prime}, r^{\prime}\right]\right\rangle: q<r=q^{\prime}<r^{\prime}, q, r, q^{\prime}, r^{\prime} \in \mathbb{R}\right\}$ \\
\hline overlaps: $\left\{\left\langle[q, r],\left[q^{\prime}, r^{\prime}\right]\right\rangle: q<q^{\prime}<r<r^{\prime}, q, r, q^{\prime}, r^{\prime} \in \mathbb{R}\right\}$ \\
\hline starts: $\left\{\left\langle[q, r],\left[q^{\prime}, r^{\prime}\right]\right\rangle: q=q^{\prime}<r<r^{\prime}, q, r, q^{\prime}, r^{\prime} \in \mathbb{R}\right\}$ \\
\hline ends: $\left\{\left\langle[q, r],\left[q^{\prime}, r^{\prime}\right]\right\rangle: q^{\prime}<q<r=r^{\prime}, q, r, q^{\prime}, r^{\prime} \in \mathbb{R}\right\}$ \\
\hline contains: $\left\{\left\langle[q, r],\left[q^{\prime}, r^{\prime}\right]\right\rangle: q<q^{\prime}<r^{\prime}<r, q, r, q^{\prime}, r^{\prime} \in \mathbb{R}\right\}$ \\
\hline
\end{tabular}

Figure 1: Interval relations

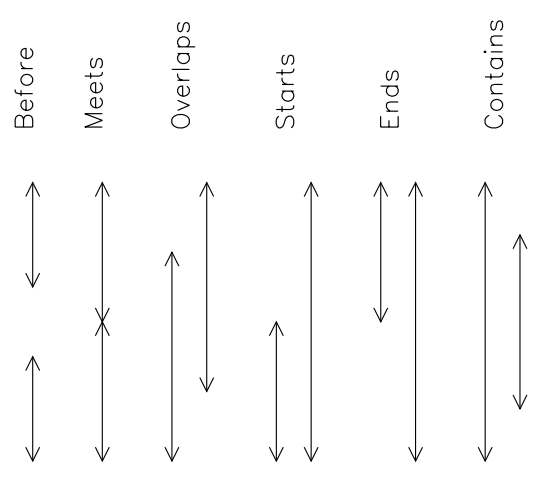

If we want to extend the time interval relations to, say, two dimensional Euclidean space, a natural domain to choose is the set $\mathfrak{C}$ of closed disks. In space, we do not have the direction of the real line any more, and thus, for example, we cannot distinguish between the "starts" and the "ends" relations, and between the "before" relation and its converse. In this spirit, we obtain the spatial relations which are defined in Table 3, and pictured in Figure 2. There, $\operatorname{int}(a)$ is the topological interior of $a$, and

Table 3: Circle relations

\begin{tabular}{|ll|}
\hline Disconnected (DC) & $:\{\langle a, b\rangle: a \cap b=\emptyset\}$ \\
Externally connected (EC) & $:\{\langle a, b\rangle: a \cap b \neq \emptyset$, int $(a \cap b)=\emptyset\}$ \\
Partial overlap (PO) & $:\{\langle a, b\rangle: a \nsubseteq b, b \nsubseteq a, \operatorname{int}(a \cap b) \neq \emptyset\}$ \\
Tangential proper part (TPP) & $:\{\langle a, b\rangle: a \subsetneq b, \operatorname{Fr}(a) \cap \operatorname{Fr}(b) \neq \emptyset\}$ \\
Nontangential proper part (NTPP) $:\{\langle a, b\rangle: a \subseteq \operatorname{int}(b)\}$. \\
\hline
\end{tabular}

$\operatorname{Fr}(a)$ its boundary, i.e. $\operatorname{Fr}(a)=a \cap-i n t(a)$. We note that $D C, E C$, and $P O$ are symmetric, while $T P P$ and $N T P P$ are not; this gives us the additional circle relations $T P P^{\triangleleft}$ and $N T P P^{\triangleleft}$. Along with $1^{\prime}$, they are the atoms of a BRA $\mathcal{C}_{c}$ on $\mathfrak{C}$ whose composition is given in Table 4 (Düntsch et al., 1999). $\mathcal{C}_{c}$ is isomorphic to the subalgebra of $\mathcal{I}$ generated by the union of the "before" relation and its converse, but its circle representation cannot be embedded into any representation of $\mathcal{I}$ : Consider the square and its diagonals in Figure 3 on the next page, and label the sides of the square with $P O$ and its 
Figure 2: Circle relations

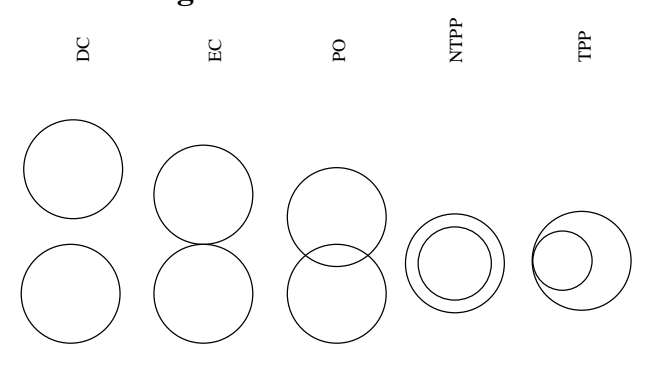

Table 4: Closed circle algebra $\mathcal{C}_{c}$

\begin{tabular}{|c|c|c|c|c|c|c|c|}
\hline$\circ$ & $T P P$ & $T P P^{\sim}$ & $N T P P$ & $N T P P^{\smile}$ & $P O$ & $E C$ & $D C$ \\
\hline$T P P$ & $P P$ & $-\left(N T P P \cup N T P P^{\cup}\right)$ & $N T P P$ & $-P$ & $-P^{凶}$ & $E C, D C$ & $\overline{D C}$ \\
\hline$T P P^{\cup}$ & $1^{\prime}, T P P, T P P^{\triangleleft}, P O$ & $P P^{\sim}$ & $P P^{\backsim}, P O$ & $N T P P^{\cup}$ & $P P^{\backsim}, P O$ & $\overline{P P^{\smile}, P O, E C}$ & $-P$ \\
\hline$N T P P$ & $N T P P$ & $-P^{\backsim}$ & $N T P P$ & 1 & $-P^{\sim}$ & $D C$ & $D C$ \\
\hline$N T P P^{\vee}$ & $P P^{\cup}, P O$ & $N T P P^{\cup}$ & $-(E C \cup D C)$ & $N T P P^{\checkmark}$ & $P P^{\cup}, P O$ & $P P^{\cup}, P O$ & $-P$ \\
\hline$P O$ & $P P, P O$ & $-P$ & $P P, P O$ & $-P$ & $\overline{1}$ & $-P$ & $-P$ \\
\hline$E C$ & $P P, P O, E C$ & $E C \cup D C$ & $P P, P O$ & $D C$ & $-P^{\backsim}$ & $-\left(N T P P \cup N T P P^{Y}\right)$ & $-P$ \\
\hline$D C$ & $-P^{\sim}$ & $D C$ & $-P^{\sim}$ & $D C$ & $-P^{\sim}$ & $-P^{\sim}$ & 1 \\
\hline
\end{tabular}

diagonals with $D C$. This network cannot be satisfied in any representation of $\mathcal{I}$ (Ladkin \& Maddux, 1994), but it can be satisfied in the closed circle algebra.

Figure 3: Satisfiable circle network

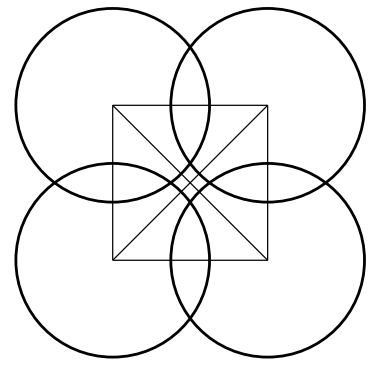

\section{Contact relation algebras}

Contact relations first arose in the works of de Laguna (1922) and Whitehead (1929). Subsequently, Clarke (1981) used a "connection" relation to extend the mereological part of the calculus of Leśniewski. It is nowadays customary to talk about "contact" instead of "connection" relations (just as de Laguna did), in order to avoid confusion with the unary topological predicate "connected". Contact relations are the backbone of current qualitative spatial reasoning (Asher \& Vieu, 1995, Cohn, 1997, Cohn et al., 1997, Gotts, 1996b). 
$C \in \operatorname{Rel}(U)$ is called a contact relation if

$C$ is reflexive and symmetric

$C x=C y \Longleftrightarrow x=y$.

It was shown by Düntsch et al. (1999) that the extensionality axiom (4.2) can be replaced by the RA term equation

$$
C \backslash C \text { is a partial order. }
$$

In the sequel, we will write $P$ for $C \backslash C$, and set $P P=P \cap-1^{\prime}$.

A contact relation algebra (CRA) is an RA which is generated by a non-identity contact relation. For a first example, consider the closed circle algebra $\mathcal{C}_{c}$ of Table 4 on the preceding page: If we set

$$
C \stackrel{\text { def }}{=} 1^{\prime} \cup T P P \cup T P P^{\cup} \cup N T P P \cup N T P P^{\sim} \cup P O \cup E C,
$$

then $P=C \backslash C=1^{\prime} \cup T P P \cup N T P P$ is set inclusion, and $C$ generates $C_{c}$.

If $\mathfrak{C}_{o}$ is the set of all open circles in the Euclidean plane, and $a C b \stackrel{\text { def }}{\Longleftrightarrow} a \cap b \neq \emptyset$ for $a, b \in \mathfrak{C}_{o}$, then the BRA $\mathcal{C}_{o}$ generated by $C$ has the composition given in Table 1 on page 4 with $C=1^{\prime} \cup P P \cup P P^{\cup} \cup P O$. This algebra is also known as the containment algebra (Ladkin \& Maddux, 1994); it is isomorphic to the subalgebra of $\mathcal{C}_{c}$ generated by $P$, and isomorphic to the subalgebra of $\mathcal{I}$ generated by the union of "precedes" and "meets" and their converses. The interval algebra $\mathcal{I}$ itself, however, is not a CRA.

In the rest of this section we will present several small CRAs from Düntsch et al. (1999). This will show that CRAs do not only arise from spatial contexts. First, however, we want to express the circle relations of Table 3 by relation algebraic terms obtained from $C$ :

$$
\begin{aligned}
O & =P^{\sim} \circ P & & \text { overlap } \\
P O & =O \cdot-\left(P+P^{\sim}\right) & & \text { partial overlap } \\
E C & =C \cdot-O & & \text { external contact } \\
T P P & =P P \cdot(E C \circ E C) & & \text { tangential proper part } \\
N T P P & =P P \cdot-T P P & & \text { non-tangential proper part } \\
D C & =-C & & \text { disconnected }
\end{aligned}
$$

We will also set

$$
\begin{aligned}
& C P=P \cup P^{\sim}, \quad \text { comparable } \\
& D R=-O \quad \text { discrete. }
\end{aligned}
$$

We will keep these definitions throughout the rest of the paper. 
Figure 4: An ordering for $\mathcal{N}_{1}$

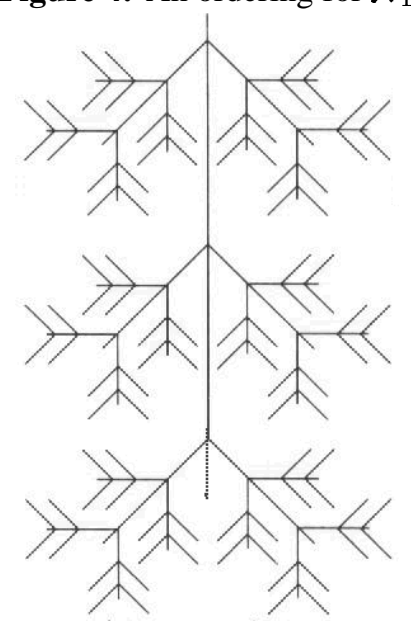

Table 5: The algebra $\mathcal{N}_{1}$

\begin{tabular}{|c||c|c|c|}
\hline$\circ$ & $P P$ & $P P^{\vee}$ & $D C$ \\
\hline \hline$P P$ & $P P$ & 1 & $D C$ \\
\hline$P P^{\vee}$ & $-D C$ & $P P^{\vee}$ & $P P^{\vee}, D C$ \\
\hline$D C$ & $P P, D C$ & $D C$ & 1 \\
\hline
\end{tabular}

Even though a contact relation $C$ need not be equal to $O$, it will always contain $O$ (Düntsch et al., 1999).

The smallest CRA is the algebra known as $\mathcal{N}_{1}$ (Comer, 1983); it has four atoms, and its composition is given in Table 5. Clearly, $C=P^{\cup} \circ P=P \cup P^{\cup}$ is symmetric and reflexive, and $P$ is a partial order. Finally,

$$
C \backslash C=-(C \circ D C)=-\left(\left(P \cup P^{\cup}\right) \circ D C\right)=-\left(D C \cup P^{\cup}\right)=P .
$$

The first concrete representation of $\mathcal{N}_{1}$ was given by (Düntsch, 1991). A picture of the order derived from a slightly different representation of $\mathcal{N}_{1}$, given in Andréka et al. (1994), is shown in Figure 4. There, $P$ is a fractal-like structure with a copy of $\mathbb{Q}$ as its stem, and branching at each point into two copies of $\mathbb{Q}$.

In $\mathcal{N}_{1}$, we have $C=O$. Contrary to this, the algebra $\mathcal{C}_{c}$ satisfies

$$
O=P^{\triangleleft} \circ P=P \cup P O
$$

and thus,

$$
E C=C \cap-O \neq \emptyset
$$

A minimal example for this situation should have the five atoms $1^{\prime}, P P, P P^{\sim}, E C$, and $D C$. Such a CRA is given in Table 6; we note that in this algebra $O$ is not a contact relation. A representation of $\mathcal{S}_{0}$ is as follows: Let

$$
\begin{aligned}
& S=\left\{\frac{a}{3^{k}}: a \lesseqgtr 3^{k}, a \text { odd, } k=1,2,3, \ldots\right\} \\
& T=\left\{\frac{a}{3^{k}}: 0 \lesseqgtr a \lesseqgtr 3^{k}, a \text { even, } k=1,2,3, \ldots\right\} .
\end{aligned}
$$


Table 6: The algebra $\mathcal{S}_{0}$

\begin{tabular}{|c|c|c|c|c|}
\hline 0 & $P P$ & $P P^{\sim}$ & $E C$ & $D C$ \\
\hline$P P$ & $P P$ & $P P, P P^{\cup}, 1^{\prime}$ & $E C, D C$ & $D C$ \\
\hline$P P^{\vee}$ & $P P, P P^{\triangleleft}, 1^{\prime}$ & $P P^{\sim}$ & $E C$ & $E C, D C$ \\
\hline$E C$ & $E C$ & $E C, D C$ & $P P, P P^{\vee}, 1^{\prime}$ & $P P^{\checkmark}$ \\
\hline$D C$ & $E C, D C$ & $D C$ & $P P$ & $P P, P P^{\triangleleft}, 1^{\prime}$ \\
\hline
\end{tabular}

Figure 5: Tangent circles

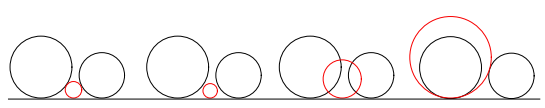

Table 7: The tangent closed circle algebra $\mathcal{T}_{c}$

\begin{tabular}{|c||c|c|c|c|}
\hline \hline$\circ$ & $P P$ & $P P^{\sim}$ & $P O$ & $D C$ \\
\hline \hline$P P$ & $P P$ & $C P$ & $-C P$ & $D C$ \\
\hline$P P^{\sim}$ & $C P$ & $P P^{\sim}$ & $P O$ & $-C P$ \\
\hline$P O$ & $P O$ & $-C P$ & $V$ & $-P$ \\
\hline$D C$ & $-C P$ & $D C$ & $-P^{\sim}$ & $V$ \\
\hline
\end{tabular}

It is not hard to see that

$$
S \cap T=\emptyset, S, T \cong \mathbb{Q},
$$

$S$ and $T$ are dense in each other,

$x \in S \Rightarrow x=\inf \{y \in T: x \lessgtr y\}=\sup \{y \in T: y \lessgtr x\}$,

$x \in T \Rightarrow x=\inf \{y \in S: x \lesseqgtr y\}=\sup \{y \in S: y \lesseqgtr x\}$,

$x \in S \Longleftrightarrow 1-x \in T$.

Now, we let $\left\langle S_{0}, \leq\right\rangle,\left\langle S_{1}, \leq\right\rangle$ be two disjoint copies of $\langle S, \leq\rangle, U=S_{0} \cup S_{1}$, and let $P$ be extension of the orders on the $S_{i}$ to $U$. Furthermore,

$$
\begin{aligned}
& x E C y \Longleftrightarrow x \in S_{i}, y \in S_{i+1} \text { and } 1-x \lessgtr y, \\
& x D C y \Longleftrightarrow x \in S_{i}, y \in S_{i+1} \text { and } 1-x \supsetneqq y .
\end{aligned}
$$

Here, $i \in\{0,1\}$, and addition is mod 2. The RA generated by $C=P \cup P^{\cup} \cup E C$ is just $\mathcal{S}_{0}$.

Finally, we present two CRAs which arise from tangent circle orders. These structures are studied in the field of preference relations (Abbas \& Vincke, 1994, Fodor \& Roubens, 1997), and the CRAs were first presented by Düntsch \& Roubens (1999). Let $\mathfrak{C}_{c}^{t}$ be the set of all open circles in the Euclidean plane which are tangent to the $x$-axis from above; an example is pictured in Figure 5. If $a C b \stackrel{\text { def }}{\Longleftrightarrow}$ $a \cap b \neq \emptyset$, then $C$ is a contact relation with $P$ being set inclusion. The CRA generated by $C$ is given in Table 7. We observe that, unlike in the closed circle algebra $\mathcal{C}_{c}, C$ loses the ability to express that two circles are tangential to each other. It is therefore somewhat surprising, that in the domain $\mathfrak{C}_{o}^{t}$ of open circles tangent to the $x$-axis, tangentiality is RA expressible: Suppose that $a C b \Longleftrightarrow a \cap b \neq \emptyset$ in $\mathfrak{C}_{o}^{t}$. It is not hard to show that $C$ is a contact relation on $\mathfrak{C}_{o}^{t}$ with $P$ being set inclusion. Let $a N T D b$ 
iff $c l(a) \cap c l(b)=\emptyset$. Observe that $N T D \subsetneq D C$, and set $T D \stackrel{\text { def }}{=} D C \cap-N T D$. Then, $a T D b$ iff $a$ and $b$ are tangential to each other. It can now be shown that

$$
P P \circ D C=N T D
$$

and thus, $T D$ and $N T D$ are RA definable. The composition of the CRA generated by $C$ on $\mathfrak{C}_{o}^{t}$ is given in Table 8.

Table 8: The tangent open circle algebra $\mathcal{T}_{o}$

\begin{tabular}{|c||c|c|c|c|c|}
\hline \hline$\circ$ & $P P$ & $P P^{\sim}$ & $P O$ & $T D$ & $N T D$ \\
\hline \hline$P P$ & $P P$ & $C P$ & $-C P$ & $N T D$ & $N T D$ \\
\hline$P P^{\sim}$ & $C P$ & $P P^{\triangleleft}$ & $P O$ & $P O$ & $P O, D C$ \\
\hline$P O$ & $P O$ & $-C P$ & $V$ & $P P^{\sim}, P O, D C$ & $P P^{\sim}, P O, D C$ \\
\hline$T D$ & $P O$ & $N T D$ & $P P, P O, D C$ & $-\left(P P \cup P P^{\triangleleft}\right)$ & $-P$ \\
\hline$N T D$ & $P O, D C$ & $N T D$ & $P P, P O, D C$ & $-P^{\sim}$ & $V$ \\
\hline
\end{tabular}

Note that $\mathcal{T}_{c}$ is isomorphic to the subalgebra of $\mathcal{T}_{o}$ generated by $C^{\prime}=C \cup T D$. Obviously,

$$
a C^{\prime} b \Longleftrightarrow \operatorname{cl}(a) \cap \operatorname{cl}(b) \neq \emptyset
$$

\section{Mereological structures}

The basic relation between individuals $x, y$ in Leśniewski's mereology is

$$
x \text { is an ingredient of } y \text {, }
$$

which we write as $x$ ingr $y$. From ingr, several other relations are defined:

$$
\begin{array}{rlrl}
x \operatorname{pt} y & \Longleftrightarrow x \operatorname{ingr} y \text { and not } x=y & \text { "part of" } \\
x \text { ov } y & \Longleftrightarrow(\exists z)[z \text { ingr } x \text { and } z \text { ingr } y] & \text { "overlaps" } \\
x \operatorname{extr} y & \Longleftrightarrow(\forall z)[z \text { ingr } x \Rightarrow \neg z \text { ingr } y] & & \text { "discrete" }
\end{array}
$$

In RA terms, these become

$$
\begin{aligned}
\mathrm{pt} & =\operatorname{ingr} \cap-1^{\prime} \\
\mathrm{ov} & =\operatorname{ingr}{ }^{`} \circ \text { ingr } \\
\text { extr } & =-\mathrm{ov} .
\end{aligned}
$$


There are two relational axioms:

$$
x \text { ingr } y \Longleftrightarrow(\forall z)[z \text { ov } x \Rightarrow z \text { ov } y]
$$

ingr is antisymmetric.

(5.7) together with (2.4) implies that

$$
\text { ingr }=\text { ov } \backslash \text { ov }
$$

and now, Lemma 2.1 tells us that ingr is reflexive and transitive, hence, with (5.8), it is a partial order. We can, alternatively, use ov as the basis relation, define ingr $\stackrel{\text { def }}{=}$ ov $\backslash$ ov, and take as axioms

$$
\text { ov is reflexive and symmetric, }
$$

ingr is antisymmetric.

These are exactly the axioms of a contact relation. If we rename ingr to $P$, pt to $P P$ and ov to $O$, then we are in accordance with our earlier terminology, and $O$ is a contact relation $C$ with the additional property that

$$
C=P^{\sim} \circ P .
$$

In $\mathrm{CM}, C$ is definable by $P$, which is usually not true in the more general case.

The other part of mereology is "sum formation" or "fusion", which we define here according to Clarke (1981), which, in the presence of (5.12), is equivalent to Leśniewski's definition. If $X$ is a collection of objects and $C$ a contact relation, then

$$
x=\sum X \stackrel{\text { def }}{\Longleftrightarrow}(\forall y)[x C y \Longleftrightarrow(\exists z \in X) y C z] .
$$

This is read as $x$ is the fusion of $X$. Now, a model of mereology is a structure $\mathfrak{M}=\left\langle U, C, \sum\right\rangle$ such that $C$ is a contact relation, and

$$
\text { For each nonempty } X \subseteq U \text { the fusion } \sum X \text { exists. }
$$

Note that this definition is not first order. If $X$ in (5.14) is finite, we speak of a weak model of mereology. If $C$ additionally satisfies (5.12), then $\mathfrak{M}$ is called a model of classical mereology.

In models of mereology, one can define the additional following operations:

$$
\begin{aligned}
1 & =\sum\{x: x C x\} & & \text { Universal element } \\
x^{*} & =\sum\{y: y(-C) x\} & & \text { Complement } \\
\prod X & =\sum\{z: z P x \text { for all } x \in X\} & & \text { Product }
\end{aligned}
$$

Observe that ${ }^{*}$ and $\prod$ are partial operations. 
The structures arising arising from Lesniewśki's classical mereology are the complete Boolean algebras with the 0 element removed, cf. Tarski (1935), p. 16f; the part - of relation $P$ is the Boolean ordering, and

$$
x C y \Longleftrightarrow x \not \leq-y .
$$

If $B$ is an atomless complete Boolean algebra, then the extreme elements 0,1 are relationally definable; thus, we are only interested in the behaviour of the contact relation and its derivatives on the set $U=B \cap-\{0,1\}$. Since $P$ is the basic relation of classical mereology, there is only one RA associated to $\mathrm{CM}$, when the Boolean algebra is atomless.

In addition to the relations defined by (4.5) - (4.10), we define the following relations:

$$
\begin{aligned}
T & =-\left(P \circ P^{\cup}\right) & & =\{\langle x, z\rangle: x+z=1\} \\
P O N & =P O \cap T & & \{\langle x, z\rangle: x(-C P) z, x \cdot z \neq 0, x+z \neq 1\} \\
P O D & =P O \cap-T & & =\{\langle x, z\rangle: x(-C P) z, x \cdot z \neq 0, x+z=1\} \\
D N & =D C \cap-T & & =\{\langle x, z\rangle: x \cdot z=0, x+z \neq 1\} \\
D D & =D C \cap T & & =\{\langle x, z\rangle: x \cdot z=0, x+z=1\}
\end{aligned}
$$

\begin{tabular}{|c|c|c|c|c|c|c|}
\hline \multirow{2}{*}{$\circ$} & \multicolumn{4}{|c|}{$\bar{O}$} & \multicolumn{2}{|l|}{$D$} \\
\hline & $\overline{P P}$ & $\overline{P P^{\sim}}$ & $P O N$ & $P O D$ & $\overline{D N}$ & $\overline{D D}$ \\
\hline$P P$ & $\overline{P P}$ & $-(P O D \cup D D)$ & $\overline{P P P, P O N, D N}$ & $\overline{P P, P O, D}$ & $\overline{D N}$ & $\overline{D N}$ \\
\hline$P P^{\sim}$ & $1^{\prime}, O$ & $P P^{\sim}$ & $P P^{\sim}, P O$ & $P O D$ & $P P^{\sim}, P O, D$ & $P O D$ \\
\hline$P O N$ & $P P, P O$ & $P P^{\sim}, P O N, D N$ & 1 & $P P, P O$ & $P P^{\sim}, P O N, D N$ & $P O N$ \\
\hline$P O D$ & $P O D$ & $P P^{\sim}, P O, D$ & $P P^{\sim}, P O$ & $1^{\prime}, O$ & $P P^{\sim}$ & $P P^{\sim}$ \\
\hline$D N$ & $P P, P O, D$ & $D N$ & $P P, P O N, D N$ & $P P$ & $-(P O D \cup D D)$ & $P P$ \\
\hline$\overline{D D}$ & $P O D$ & $D N$ & $P O N$ & $\overline{P P}$ & $P P^{\sim}$ & $1^{\prime}$ \\
\hline
\end{tabular}

The composition of the CRA $\mathcal{G}$ generated by $P$ is given in Table 9 .

Table 9: The algebra $\mathcal{G}$

In the algebra $\mathcal{G}$, there are two possibilities to define a contact relation: We can take either $C=O$ or $C=O \cup D D$. In both cases, $P=C \backslash C$. In the first case, (5.12) is also fulfilled, so that we obtain a model of classical mereology. If $C=O \cup D D$, then we do not obtain a model of mereology, since in such models, a region is never in contact with its complement. At any rate, whenever a CRA generated by $C$ assumes an underlying atomless Boolean algebra with the Boolean ordering as the "part - of" relation (such as the RCC discussed below), then the relations of $\mathcal{G}$ must be present, and $O \subseteq C$.

The algebraic part of a model of mereology need not be a Boolean algebra without a smallest element. Biacino \& Gerla (1991) have shown the following:

Proposition 5.1. If $\langle L,+, \cdot,-\rangle$ is a complete orthocomplemented lattice, then

$$
x C y \Longleftrightarrow x \not \leq-y
$$


Figure 6: An ordering for $\mathcal{S}_{1}$

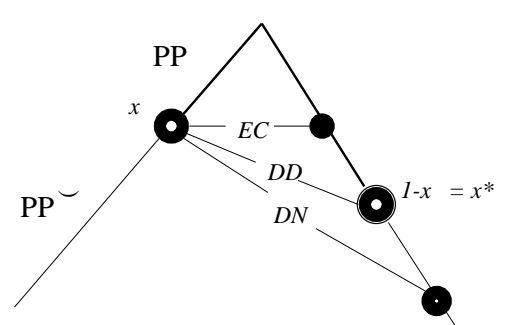

Table 10: The scale algebra $\mathcal{S}_{1}$

\begin{tabular}{|c||c|c|c|c|c|}
\hline$\circ$ & $P P$ & $P P^{\sim}$ & $E C$ & $D N$ & $D D$ \\
\hline \hline$P P$ & $P P$ & $C P$ & $-C P$ & $D N$ & $D N$ \\
\hline$P P^{\sim}$ & $C P$ & $P P^{\sim}$ & $E C$ & $E C, D C$ & $E C$ \\
\hline$E C$ & $E C$ & $-C P$ & $C P$ & $P P^{\sim}$ & $P P^{\sim}$ \\
\hline$D N$ & $E C, D C$ & $D N$ & $P P$ & $C P$ & $P P$ \\
\hline$D D$ & $E C$ & $D N$ & $P P$ & $P P^{\sim}$ & $1^{\prime}$ \\
\hline
\end{tabular}

defines a contact relation, and the fusion is just the lattice join. Conversely, if $\left\langle U, C, \sum\right\rangle$ is a model of mereology, we let $U^{\prime}=U \cup\{0\}$, where $0 \notin U$. Then, $\left\langle U^{\prime}, C, \sum\right\rangle$ is a complete orthocomplemented lattice with the lattice join being the fusion, and the other operations given by (5.16) and (5.17), extended by $\prod X=0$ whenever $\prod X$ does not exist in $U$, and $0^{*}=1,1^{*}=0$.

Any CRA $A$ for mereology must split $D C$ into $D D$ and $D N$. If $A$ is a not a model of classical mereology, i.e. if $C \neq O$, then $E C=C \cap-O \neq \emptyset$. A minimal model for this situation is as follows: Let $E_{0}, E_{1}$ be two copies of the real interval $(0,1)$ ordered as usual by $\leq$, and set $E=E_{0} \cup E_{1}, E^{+}=$ $E \cup\{1\}$. Order $E^{+}$by

$$
x P y \Longleftrightarrow x, y \in E_{i} \text { and } x \leq y \text {, or } \mathrm{y}=1 .
$$

In the following, addition is modulo 2. We let $m: E \rightarrow E$ be defined in such a way that, if $x \in E_{i}$, then $m(x)$ is the value of $x$ in $E_{i+1}$. Now, the relation $C$ defined on $E$ by

$$
\langle x, y\rangle \in C \Longleftrightarrow x \not \leq(1-m(x))
$$

defines a contact relation, and

$$
\begin{aligned}
P P & =\lessgtr \\
O & =P^{\sim} \circ P=P+P^{\triangleleft}+1^{\prime}, \\
E C & =C \backslash O=\{\langle x, y\rangle: y \supsetneqq 1-m(x)\}, \\
D D & =-\left[\left(-P^{\triangleleft} \circ D C\right) \cup\left(P^{\sim} \circ C\right)\right]=\{\langle x, y\rangle: y=1-m(x)\}, \\
D N & =D C \cap-D D=\{\langle x, y\rangle: y \lessgtr 1-m(x)\}
\end{aligned}
$$

The composition of the RA $\mathcal{S}_{1}$ generated by $C$ is given in Table 10 . We call $\mathcal{S}_{1}$ a scale algebra, since $x$ is related to its complement like a scale, as indicated in Figure 6. As indicated by the lines, the element $x$ in the left copy is linked to $1-x$ in the right copy by $D D$, to anything above $1-x$ in the right copy by $E C$, to anything below $1-x$ in the right copy by $D N$.

Our final example of this section exhibits a model of mereology where $E C$ splits according to whether $x+y=1$ or not. Let $S_{i}, i<4$ be disjoint copies of the rational interval $(0,1)$. The mapping $m$ is 
Figure 7: An ordering for $\mathcal{S}_{2}$

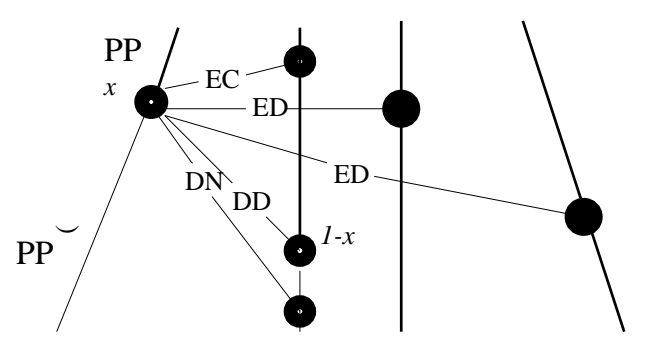

defined from

$$
m: \begin{cases}S_{0} & \rightarrow S_{1}, \\ S_{1} & \rightarrow S_{0}, \\ S_{2} & \rightarrow S_{3} \\ S_{3} & \rightarrow S_{2}\end{cases}
$$

and $m$ puts $x \in(0,1)$ onto its twin in the other component. We now define

$$
\begin{aligned}
& x P P y \Longleftrightarrow x, y \in S_{i} \text { and } x \lessgtr y, \\
& x D D y \Longleftrightarrow y=1-m(x), \\
& x E N y \Longleftrightarrow 1-m(x) \lessgtr y, \\
& x D N y \Longleftrightarrow 1-m(x) \gtrless y, \\
& x E D y \Longleftrightarrow y \text { is in a component different from that of } x \text { or } D D(x) .
\end{aligned}
$$

\begin{tabular}{|c|c|c|c|c|c|c|}
\hline o & $P P$ & $\breve{P} P^{\sim}$ & $E N^{2}$ & $E D$ & $D N$ & $D D$ \\
\hline$P P$ & $P P$ & $P P, P P^{\backsim}, 1^{\prime}$ & $E N, D C$ & $E D$ & $D N$ & $D N$ \\
\hline$P P^{\smile}$ & $P P, P P^{\smile}, 1^{\prime}$ & $P P^{\sim}$ & $E N$ & $E D$ & $E N, D C$ & $E N$ \\
\hline$E N$ & $E N$ & $E N, D C$ & $P P, P P^{\smile}, 1^{\prime}$ & $E D$ & $P^{\checkmark}$ & $P^{\checkmark}$ \\
\hline$E D$ & $E D$ & $E D$ & $E D$ & $-E D$ & $E D$ & $E D$ \\
\hline$D N$ & $E N, D C$ & $D N$ & $P P$ & $E D$ & $P P, P P^{\triangleleft}, 1^{\prime}$ & $P P$ \\
\hline$D D$ & $E N$ & $D N$ & $P P$ & $E D$ & $P P^{\sim}$ & $1^{\prime}$ \\
\hline
\end{tabular}

If $C=-(D N \cup D D)$, then $\mathcal{S}_{2}$ is isomorphic to the algebra generated by $C$. We have not been able to find an intuitive spatial explanation of this situation. The composition of $\mathcal{S}_{2}$ is shown in Table 11, and an indication of the atoms of $\mathcal{S}_{2}$ is given in Fig. 7.

Table 11: Algebra $S_{2}$ with complement and split EC

We can also have $E D \circ E D=1$; in this case, we need (at least) six components, and, otherwise, use the same definitions as for $S_{2}$. Figure 7 is to be interpreted similarly to Figure 6: In addition, $x$ in the leftmost copy is related to any $y$ in the two rightmost copies by $E D$. 


\section{The region connection calculus}

The Region Connection Calculus (RCC) was introduced by Randell et al. (1992) as a vehicle for reasoning about spatial phenomena, and has since received some prominence. It uses a contact relation $C$ which fulfils Clarke's axioms (4.1) and (4.2).

A model for the RCC consists of a base set $U=R \cup N$, where $R, N$ are disjoint, a distinguished $u \in R$, a unary operation $-: R_{0} \rightarrow R_{0}$, where $R_{0} \stackrel{\text { def }}{=} R \backslash\{u\}$, a binary operation $+: R \times R \rightarrow R$, another binary operation $\cdot: R \times R \rightarrow R \cup N$, and a binary relation $C$ on $R$.

The RCC axioms are as follows:

RCC 1. $(\forall x \in R) x C x$

RCC 2. $(\forall x, y \in R)[x C y \Rightarrow y C x]$

RCC 3. $(\forall x \in R) x C u$

RCC 4. $\left(\forall x \in R, y \in R_{0}\right)$,

(a) $\langle x,-y\rangle \in C \Longleftrightarrow \neg x N T P P y$

(b) $\langle x,-y\rangle \in O \Longleftrightarrow \neg x P y$

RCC 5. $(\forall x, y, z \in R)[\langle x, y+z\rangle \in C \Longleftrightarrow x C y$ or $x C z$

RCC 6. $(\forall x, y, z \in R)[\langle x, y \cdot z\rangle \in C \Longleftrightarrow(\exists w \in R)(w P y$ and $w P z$ and $x C w)]$

RCC 7. $(\forall x, y \in R)[x \cdot y \in R \Longleftrightarrow x O y]$

RCC 8. If $x P y$ and $y P x$, then $x=y$.

We shall in the sequel assume without loss of generality that $N=\{0\}$, and, to avoid trivialities, we suppose that $|R| \geq 2$. Axioms RCC 1 , RCC 2, RCC 5 and RCC 8 show that $\langle R, C,+\rangle$ is a weak model of mereology. It is, however, not a model of mereology in the sense of Section 5, since it has a different definition of complement: In the RCC models, each proper region $x$ is connected to its complement $-x$, which is impossible in models of mereology. Still

Proposition 6.1. (Düntsch et al., 1999, Stell, 1997)

Each model of the RCC axioms is a Boolean algebra with $N=\{0\}$.

In the original RCC, the circle relations of Table 3

$$
1^{\prime}, T P P, T P P^{\sim}, N T P P, N T P P^{\sim}, P O, E C, D C
$$

are considered base relations in a system called RCC8, and the weak composition table presented by Randell et al. (1992) is shown in Table 12. 
Table 12: RCC8 weak composition table

\begin{tabular}{|c|c|c|c|c|c|c|c|}
\hline \multirow{4}{*}{${ }^{\circ} w$} & & \multicolumn{6}{|c|}{$C$} \\
\hline & \multirow{2}{*}{\multicolumn{2}{|c|}{$D R$}} & \multicolumn{5}{|c|}{$\bar{O}$} \\
\hline & & & & \multicolumn{2}{|c|}{$P P$} & \multicolumn{2}{|c|}{$P P^{\sim}$} \\
\hline & $D C$ & $E C$ & $P O$ & $T P P$ & $N T P P$ & $T P P^{\smile}$ & $N T P P^{\sim}$ \\
\hline$\overline{D C}$ & 1 & $D R, P O, P P$ & $D R, P O, P P$ & $D R, P O, P P$ & $D R, P O, P P$ & $\overline{D C}$ & $D C$ \\
\hline$E C$ & $D R, P O, P P^{\backsim}$ & $\begin{array}{l}1^{\prime}, D R, P O, \\
T P P T P P^{\sim}\end{array}$ & $D R, P O, P P$ & $E C, P O, P P$ & $P O, P P$ & $D R$ & $D C$ \\
\hline$P O$ & $D R, P O, P P^{\backsim}$ & $D R, P O, P P^{\triangleleft}$ & 1 & $P O, P P$ & $P O, P P$ & $D R, P O, P P^{\sim}$ & $D R, P O, P P^{\sim}$ \\
\hline$T P P$ & $D C$ & $\overline{D R}$ & $D R, P O, P P$ & $P P$ & $N T P P$ & $\begin{array}{l}l^{\prime}, D R, P O, \\
T P P, T P P^{\sim}\end{array}$ & $D R, P O, P P^{\sim}$ \\
\hline$N T P P$ & $D C$ & $D C$ & $D R, P O, P P$ & NTPP & $N T P P$ & $D R, P O, P P$ & 1 \\
\hline$T P P^{\sim}$ & $D R, P O, P P^{\sim}$ & $E C, P O, P P^{\sim}$ & $P O, P P^{\sim}$ & $\begin{array}{l}l^{\prime}, P O, \\
T P P, T P P^{\sim}\end{array}$ & $P O, P P$ & $P P^{\sim}$ & $N T P P^{\sim}$ \\
\hline$N T P P^{\backsim}$ & $D R, P O, P P^{\backsim}$ & $P O, P P^{\backsim}$ & $P O, P P^{\sim}$ & $P O, P P^{\sim}$ & $O \cup 1^{\prime}$ & $N T P P^{\sim}$ & $N T P P^{\backsim}$ \\
\hline
\end{tabular}

It was asked in Bennett et al. (1997), when the RCC8 table has an extensional interpretation, i.e. when it can be interpreted as the composition table of a CRA. Table 4 on page 6 shows that the closed circle algebra $\mathcal{C}_{c}$ provides such an interpretation.

Since each RCC model is a Boolean algebra $B$, we can restrict our investigations to $U \stackrel{\text { def }}{=} B \cap-\{0,1\}$. Then, as with $\mathcal{G}, P O$ splits into $P O N$ and $P O D$, and $D C$ splits into $D N$ and Boolean complement $D D$ as defined in (5.20) - (5.23). Since each region is connected to its complement, and we want to reserve $D C$ for the "disconnected" relation, we use different names as follows:

$$
\begin{aligned}
D C & \stackrel{\text { def }}{=}-C=D N \text { of (5.22) } \\
E C D & \stackrel{\text { def }}{=} D D \text { of }(5.23) \\
E C N & \stackrel{\text { def }}{=} E C \cap-E C D \Longleftrightarrow x E C y \text { and } x+y \neq u,
\end{aligned}
$$

Düntsch et al. (2001a) have shown that $E C N \circ T P P \lesseqgtr P O D$, and thus, $P O D$ splits into

$$
\begin{aligned}
& P O D Z \stackrel{\text { def }}{=} E C D \circ N T P P \\
& P O D Y \stackrel{\text { def }}{=} P O D \backslash(E C D \circ N T P P) .
\end{aligned}
$$

The resulting system, RCC11, has the weak composition given in Table 13 on the next page. For cells containing $=$, the RCC axioms together with general RA properties imply that equality holds; for cells containing $\neq$, there is a model in which the composition is strictly smaller than the cell entry. In this way, we indicate in which cells the composition may be weak, and when it is not. Indeed, it turns out that a CRA model of the RCC relations has at least 25 atoms (Düntsch et al., 2001a).

A standard RCC model is the Boolean algebra $R O(X)$ of regular open sets of a connected regular topological space $\langle X, \tau\rangle$, where for $x, y \in R O(X)$,

$$
x C y \stackrel{\text { def }}{\Longleftrightarrow} \operatorname{cl}(x) \cap \operatorname{cl}(y) \neq \emptyset
$$

(Gotts, 1996a). 


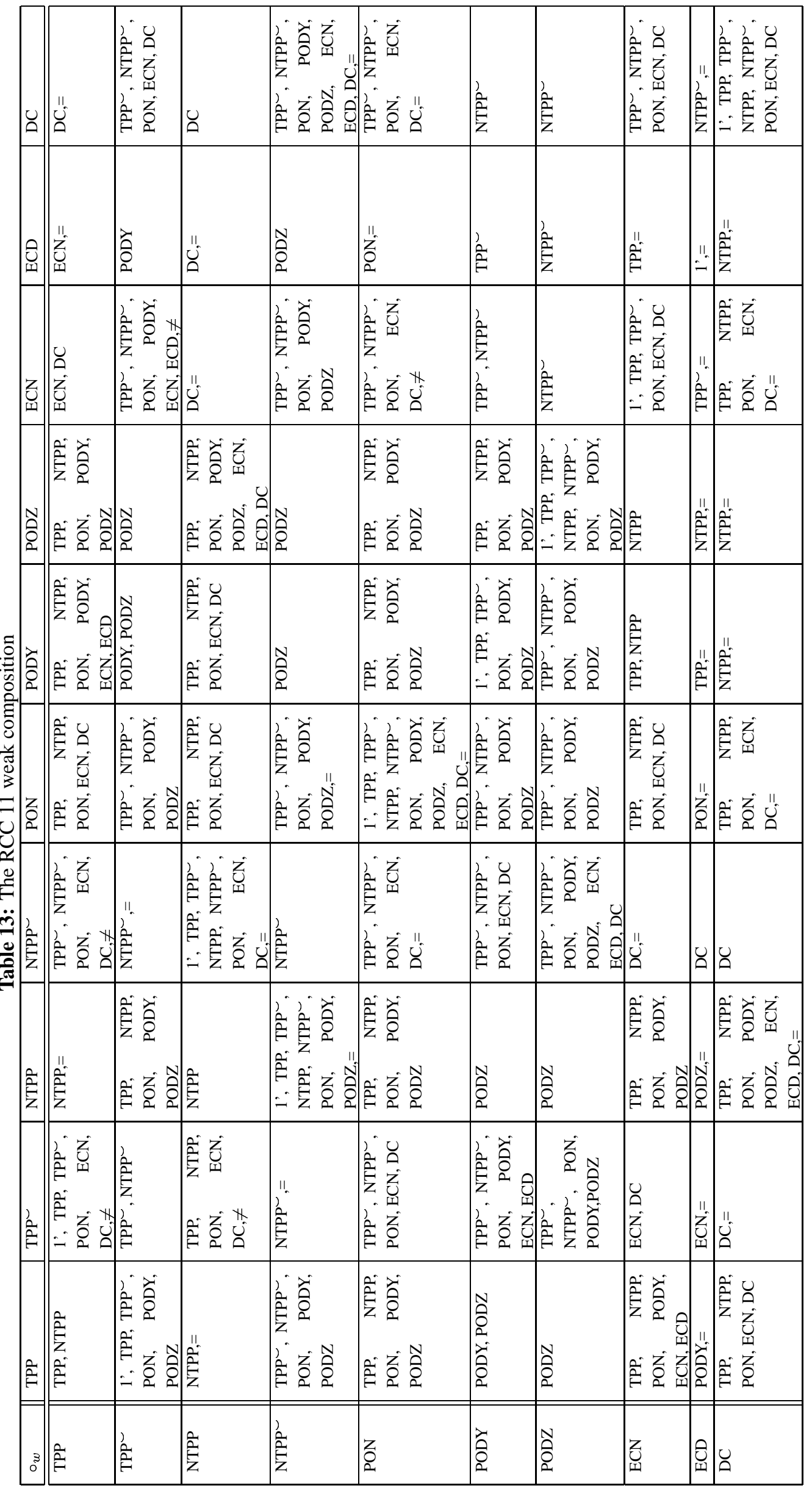


The question was raised in Bennett et al. (1997) in which situations a composition table for the CRA associated with a model of RCC is first order closed. The following necessary conditions was established by Düntsch et al. (2001b). For this, recall that a Boolean algebra $B$ is homogeneous, if every nontrivial relative algebra $B\lceil b$ is isomorphic to $B$ (see Koppelberg, 1989).

Proposition 6.2. If $A$ is a first order closed CRA obtained from an RCC model, then the underlying Boolean algebra $B$ is homogeneous.

A subset of the circle relations, namely,

$$
1^{\prime}, D R, P O, P P, P P^{\sim}
$$

has received some attention, and is usually called RCC5 (Bennett, 1997, Jonsson \& Drakengren, 1997). It arises from disregarding the split of $C$ into $O$ and $E C$, and $P P$ into $T P P$ and $N T P P$; in other words, one adds the additional axiom $C=O$.

Restricting relations to the non-extremal elements of a model, we arrive at seven relations and a weak composition table which has an extensional interpretation as the CRA $\mathcal{G}$ generated by the order of an atomless Boolean algebra.

\section{Summary and outlook}

We have given a survey of the origins and many examples of contact relations and their algebras. We have shown how spatial relations among open or closed circles are naturally obtained from the one-dimensional interval relations. "Part-of" and "contact" relations arising in mereology were the motivating factor in defining contact relation algebras (CRAs). We have given small examples for these algebras, and also explored CRAs which arise from the RCC, a well known mechanism for spatial reasoning.

There are many avenues which can be followed, and, to conclude, we present the questions raised in Düntsch et al. (1999):

- For which partial orders $P$ is there a contact relation $C$ such that $P=C \backslash C$ ? When can $C$ be chosen as $P^{\triangleleft} \circ P$ ?

- Investigate the complexity of CRAs. This is an important question, relating to the feasibility of relational reasoning in QSR (Bennett et al., 1997). There have been investigations for the interval algebra and its relatives (Hirsch, 1997, Ladkin \& Maddux, 1994, Nebel \& Bürckert, 1993), as well as for RA-like structures related to the RCC (Jonsson \& Drakengren, 1997, Renz \& Nebel, 1997, 1998). In connection with the different representations of subalgebras of the interval algebra, it is also of interest to investigate the network satisfaction problem for the given algebras and their representations (Hirsch, 1997). 
- Find the CRAs for standard ontologies of mereo-topology and their expressiveness. These include the standard model of the RCC as the collection of all nonempty regular closed sets on a regular connected spaces, as well as the polygonal algebras of Pratt \& Schoop (1998, 2000). It is shown by Düntsch et al. (2001a) that such a CRA must contain at least 25 atoms.

- Look at vagueness of spatial regions. This seems especially important for applications such as geographical information systems (Worboys, 1998). The rough relations of Comer (1993) and Düntsch (1994), or the uncertainty approach of Düntsch \& Gediga (1997) may come in useful. It should also be worthwhile to investigate the connections of rough mereology (Polkowski \& Skowron, 1996) to this problem.

\section{Acknowledgement}

I should like to thank the participants of RelMiCS 4 and its organiser, Ewa Orłowska, for providing a stimulating atmosphere from which the current work received many inspirations.

\section{References}

Abbas, M. \& Vincke, P. (1994). Tangent circle orders : Numerical representation and properties. Manuscript.

Allen, J. F. (1983). Maintaining knowledge about temporal intervals. Communications of the ACM, 26, 832-843.

Allen, J. F. (1984). Towards a general theory of action and time. Artificial Intelligence, 23, 123-154.

Andréka, H., Givant, S. \& Németi, I. (1994). The lattice of varieties of representable relation algebras. Journal of Symbolic Logic, 59, 631-661.

Asher, N. \& Vieu, L. (1995). Toward a geometry of common sense: A semantics and a complete axiomatization of mereotopology. In C. Mellish (Ed.), IJCAI 95, Proceedings of the 14th International Joint Conference on Artificial Intelligence.

Bennett, B. (1997). Logical representations for automated reasoning about spatial relationships. Doctoral dissertation, School of Computer Studies, University of Leeds.

Bennett, B. (1998). Determining consistency of topological relations. Constraints, 3, 213-225.

Bennett, B., Isli, A. \& Cohn, A. (1997). When does a composition table provide a complete and tractable proof procedure for a relational constraint language? In IJCAI 97, Proceedings of the Workshop of Spatial Reasoning.

Betti, A. (1997). Stanisław Leśniewski. http://www.fmag •unict.it/PolPhil/Lesnie/ Lesnie.html. 
Biacino, L. \& Gerla, G. (1991). Connection structures. Notre Dame Journal of Formal Logic, 32, 242-247.

Chin, L. \& Tarski, A. (1951). Distributive and modular laws in the arithmetic of relation algebras. University of California Publications in Mathematics, 1, 341-384.

Clarke, B. L. (1981). A calculus of individuals based on 'connection'. Notre Dame Journal of Formal Logic, 22, 204-218.

Cohn, A. G. (1997). Qualitative spatial representation and reasoning techniques. Research report, School of Computer Studies, University of Leeds.

Cohn, A. G., Bennett, B., Gooday, J. \& Gotts, N. M. (1997). Qualitative spatial representation and reasoning with the region connection calculus. Geoinformatica, 13, pp 1-42.

Comer, S. (1983). A remark on chromatic polygroups. Congressus Numerantium, 38, 85-95.

Comer, S. (1993). On connections between information systems, rough sets, and algebraic logic. In C. Rauszer (Ed.), Algebraic Methods in Logic and Computer Science, vol. 28 of Banach Center Publications, 117-124. Warszawa: Polish Academy of Science.

de Laguna, T. (1922). Point, line and surface as sets of solids. The Journal of Philosophy, 19, 449-461.

Düntsch, I. (1991). Small integral relation algebras generated by a partial order. Period. Math. Hungar., 23, 129-138.

Düntsch, I. (1994). Rough relation algebras. Fundamenta Informaticae, 21, 321-331.

Düntsch, I. \& Gediga, G. (1997). Relation restricted prediction analysis (Extended abstract). In A. Sydow (Ed.), Proc. 15th IMACS World Congress, Berlin, vol. 4, 619-624, Berlin. Wissenschaft und Technik Verlag.

Düntsch, I. \& Roubens, M. (1999). Tangent circle algebras. Submitted for publication.

Düntsch, I., Schmidt, G. \& Winter, M. (2001a). A necessary relation algebra for mereotopology. Studia Logica, 69, 381-409.

Düntsch, I., Wang, H. \& McCloskey, S. (1999). Relation algebras in qualitative spatial reasoning. Fundamenta Informaticae, 39, 229-248.

Düntsch, I., Wang, H. \& McCloskey, S. (2001b). A relation algebraic approach to the Region Connection Calculus. Theoretical Computer Science, 255, 63-83.

Egenhofer, M. \& Franzosa, R. (1991). Point-set topological spatial relations. International Journal of Geographic Information Systems, 5, 161-174.

Eschenbach, C. \& Heydrich, W. (1995). Classical mereology and restricted domains. International Journal of Human-Computer Studies, 43, 723-740. 
Fodor, J. \& Roubens, M. (1997). Parametrized preference structures and some geometrical interpretation. Journal of Multi-Criteria Decision Analysis, 6, 253-258.

Gotts, N. M. (1996a). An axiomatic approach to topology for spatial information systems. Research Report 96.25, School of Computer Studies, University of Leeds.

Gotts, N. M. (1996b). Topology from a single primitive relation: Defining topological properties and relations in terms of connection. Research Report 96.23, School of Computer Studies, University of Leeds.

Hirsch, R. (1997). Expressive power and complexity in algebraic logic. Journal of Logic and Computation, 7, 309-351.

Jónsson, B. (1982). Varieties of relation algebras. Algebra Universalis, 15, 273-298.

Jónsson, B. (1991). The theory of binary relations. In H. Andréka, J. D. Monk \& I. Németi (Eds.), Algebraic Logic, vol. 54 of Colloquia Mathematica Societatis János Bolyai, 245-292. Amsterdam: North Holland.

Jonsson, P. \& Drakengren, T. (1997). A complete classification of tractability in RCC-5. Journal of Artificial Intelligence Research, 6, 211-222.

Koppelberg, S. (1989). General Theory of Boolean Algebras, vol. 1 of Handbook on Boolean Algebras. North Holland.

Ladkin, P. B. \& Maddux, R. D. (1994). On binary constraint problems. Journal of the ACM, 41, 435-469.

Leonard, H. S. \& Goodman, N. (1940). The calculus of individuals and its uses. Journal of Symbolic Logic, 5, 45-55.

Leśniewski, S. (1927 - 1931). O podstawach matematyki. Przeglad Filozoficzny, 30-34.

Leśniewski, S. (1983). On the foundation of mathematics. Topoi, 2, 7-52.

Link, G. (1998). Algebraic Semantics in Language and Philosophy, vol. 74 of CSLI Lecture Notes. Stanford: Center for the Study of Language and Information.

Luschei, E. C. (1962). The Logical Systems of Leśniewski. Amsterdam: North Holland.

Lyndon, R. C. (1950). The representation of relational algebras. Annals of Mathematics (2), 51, 707-729.

Nebel, B. \& Bürckert, H.-J. (1993). Reasoning about temporal relations: A maximal tractable subclass of Allen's interval algebra. Research Report RR-93-11, German Research Center for Artificial Intelligence (DFKI), Saarbrücken, Germany. 
Nicod, J. (1924). Geometry in a sensible world. Doctoral thesis, Sorbonne, Paris. English translation in Geometry and Induction, Routledge and Kegan Paul, 1969.

Polkowski, L. \& Skowron, A. (1996). Rough mereology: A new paradigm for approximate reasoning. International Journal of Approximate Reasoning, 15, 333-365.

Pratt, I. \& Schoop, D. (1998). A complete axiom system for polygonal mereotopology of the real plane. Journal of Philosophical Logic, 27, 621-658.

Pratt, I. \& Schoop, D. (2000). Expressivity in polygonal, plane mereotopology. Journal of Symbolic Logic, 65, 822-838.

Pratt, V. R. (1990). Dynamic algebras as a well-behaved fragment of relation algebras. In Algebraic Logic and Universal Algebra in Computer Science, LNCS 425, 77-110, Ames, Iowa, June 1988. Springer-Verlag.

Randell, D. A., Cohn, A. G. \& Cui, Z. (1992). Computing transitivity tables: A challenge for automated theorem provers. In D. Kapur (Ed.), Proceedings of the 11th International Conference on Automated Deduction (CADE-11), vol. 607 of LNAI, 786-790, Saratoga Springs, NY. Springer.

Renz, J. \& Nebel, B. (1997). On the complexity of qualitative spatial reasoning: A maximal tractable fragment of the Region Connection Calculus. In IJCAI 97, Proceedings of the 15th International Joint Conference on Artificial Intelligence.

Renz, J. \& Nebel, B. (1998). Efficient methods for qualitative spatial reasoning. In H. Prade (Ed.), Proceedings of the 13th European Conference on Artificial Intelligence, ECAI'98, 562-566. Wiley.

Simons, P. (1987). Parts. A Study in Ontology. Oxford: Clarendon Press.

Stell, J. (1997). Personal communication, October 30, 1997.

Surma, S. J., Srzednicki, J. T., Barnett, D. I. \& Ricky, V. F. (Eds.) (1992). Stanisław Leśniewski: Collected works.

Tarski, A. (1935). Zur Grundlegung der Boole'schen Algebra, I. Fundamenta Mathematicae, 24, $177-198$.

Tarski, A. (1941). On the calculus of relations. Journal of Symbolic Logic, 6, 73-89.

Tarski, A. \& Givant, S. (1987). A formalization of set theory without variables, vol. 41 of Colloquium Publications. Providence: Amer. Math. Soc.

van Benthem, J. (1983). The logic of time. Reidel.

Whitehead, A. N. (1929). Process and reality. New York: MacMillan.

Worboys, M. (1998). Imprecision in finite resolution spatial data. Geoinformatica, 2, 257-280. 